\title{
Interessante Fakten zum Röntgen in der Praxis
}

\author{
M. Beyeler
}

1 SAP Schweizer Automatik Pool. Digitales Röntgen für die Arztpraxis. Schweiz Ärztezeitung 2003; 84(48):2555-6.

2 BAG. Strahlenschutzprüfung einfach per Internet. Internetprüfung für Sachverstand im Strahlenschutz. Schweiz Ärztezeitung 2004;85(1/2):21.

3 OFSP. L'examen pour les experts en radioprotection? Sur Internet, tout simplement! Examen sur Internet pour les experts en radioprotection. Bull Med Suisses 2004; 85(1/2):22.

Korrespondenz:

Markus Beyeler

WIROMA AG

Waldeggstrasse 63/1

CH-3097 Liebefeld-Bern

Tel. 0319740874

Fax 0319740870

\section{Digitales Röntgen - Spielzeug für den Computerfreak oder echte Innovation?}

Im Heft Nr. 48/2003 sind Technologien, Verfahren und Vorteile hinlänglich beschrieben worden [1]. Hier geht es um Entscheidungshilfen für den innovations- und investitionsfreudigen Arzt.

Wie bereits bei der revolutionären Eroberung der Bürowelt durch den Computer, werden auch beim digitalen Röntgen die Preise erfahrungsgemäss fallen. Eben wurden die ersten Anlagen für den Hausarzt noch für über Fr. 200000.- gehandelt. Heute mutmassen Spezialisten aber, dass der Preis nächstens unter Fr. 100000.- fallen könnte, und dies für eine Komplettanlage! Wir können uns dieser optimistischen Meinung anschliessen.

Die im Moment gängigste Technologie mit der Speicherfolie dürfte wohl noch für einige Zeit vielen Ansprüchen genügen. Mit grossen Anstrengungen betreiben bekannte Röntgenhersteller Entwicklung und Forschung für das Flatpanelsystem, welches zukünftig auch beim Hausarzt eingesetzt werden kann. Es ist zu erwarten, dass dieses System alle andern Technologien ablösen wird. Der Preis wird langfristig demjenigen der heutigen konventionellen Systeme sehr nahekommen.

Ist die digitale Lösung überhaupt fürs Portemonnaie des Hausarztes? Das hängt natürlich von der Anzahl Expositionen ab. Ab 700 Bilder im Jahr ist man bereits mit heutigen Digitalanlagen im «schwarzen» (rentablen) Bereich.

Auch Banken zeigen Interesse für das digitale Röntgen! Die Zukunftsvision des Geldinstitutes ist, den Kunden eine umfassendere Dienstleistung anbieten zu können. So könnten zum Beispiel Ärzte, welche bei dieser Bank bereits ein Konto eingerichtet haben, sich mittels Kreditkarte einloggen und so ihre digitalen Röntgenbilder abspeichern. Die Bank stellt damit nicht nur den Speicherplatz zur Verfügung, sondern sie übernimmt auch die sichere Archivierung der Daten.

\section{Achtung: Ab 1. Januar 2008 gelten neue Vorschriften}

Bereits vor über 10 Jahren kündigte der Gesetzgeber auf 1. Januar 2008 neue Vorschriften für die Verwendung der verschiedenen Empfindlichkeitsklassen von Film-Folien-Systemen an.

Die Röntgenkassetten sind mit Folien ausgelegt. Diese Folien haben die physikalische Eigenschaft beim Auftreffen von unsichtbarer Röntgenstrahlung, diese in Licht, welches der spektralen Empfindlichkeit des Films entspricht, umzuwandeln.

Folien mit einer Empfindlichkeit von 100 benötigen im Vergleich zu einer 400er Folie die vierfache Dosis. Das bedeutet für den Patienten $75 \%$ weniger Dosis. Diese Dosiseinsparnis ist nicht nur für den Patienten und das Röntgenpersonal von Vorteil, dadurch wird auch die Röntgenröhre weniger belastet. Das spricht für eine längere Lebensdauer der Röhre.

Dies bedingt aber, dass der Generator in der Lage sein sollte, solch kleine mAs-Produkte schalten zu können. Konnte früher eine Thoraxaufnahme z.B. mit $6 \mathrm{mAs}$ exponiert werden, so sollte zukünftig 1,5 mAs eingestellt werden. Das stellt ältere 2-Puls-Generatoren, bei welchen ein Röhrenstrom gleich/kleiner $50 \mathrm{~mA}$ nicht angewählt werden kann, vor grosse Probleme. Unter Umständen drängt sich ein Generatorersatz auf. Aber seien Sie vorsichtig. Der grösste Teil der heute installierten Röntgenanlagen in Hausarztpraxen wird diese Neuerung erfüllen. Sollte Ihnen ein Generatortausch empfohlen oder gar der alte Generator abgesprochen werden, so holen Sie sich unbedingt eine Zweitmeinung ein.

\section{Weitere Informationen}

- Sachverstand im Strahlenschutz. Das BAG bietet neu die Prüfung auch im Internet an. Davon können Ärztinnen und Ärzte, welche die Röntgenbewilligung vor dem 1. Oktober 1994 erhalten haben, profitieren [2, 3].

- Sachkunde für dosisintensives Röntgen: www. kollegium.ch informiert unter der Rubrik «Ausweise». 


\section{Röntgenkontrollen (Zustandsprüfungen und Wartungen)}

Aufgrund des allgemein guten Zustandes der Röntgeneinrichtungen in der Arztpraxis drängt sich die Frage nach einer Lockerung der Wartungsintervalle auf. Warum die Wartungen nicht alle 6 Jahre, anstatt wie heute, alle 3 Jahre durchführen?

Die qualitätssichernden Massnahmen verlängern die Lebensdauer der Röntgeneinrichtung schätzungsweise um 30\%. Demgegenüber steht der von den Röntgenfirmen geleistete Aufwand mit jährlich Fr. 900.- im Durchschnitt. Viele Firmen bieten erst noch Spezialtarife an, wenn nebst den Dienstleistungen auch das Verbrauchsmaterial geliefert werden kann.

Auch die Röntgenfirmen spüren den Kostendruck. Einerseits sank das Preisniveau in den letzten 10 Jahren für komplette Röntgenanlagen um Fr. 20000.-, andererseits stiegen die Kosten durch zusätzliche Neuerungen und Vorschriften (Zertifizierungszwang, regelmässige Eichung der Messgeräte usw.).

Immerhin prüft das Bundesamt für Gesundheit (BAG) im Moment, ob an den in ganz Europa gültigen Normen etwas geändert werden sollte. Das BAG plant, mit den in der Schweiz tätigen Fachfirmen in Verbindung zu treten. Ein Diskussionspunkt dürften die Wartungsintervalle sein.

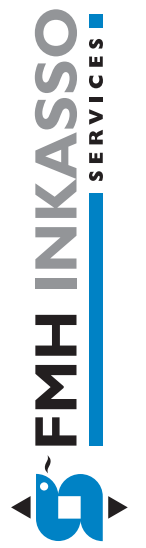

Ihr Partner, wenn Zahlungseingänge sich verzögern!

Säumige Patientinnen und Patienten? Wenn die Zahlungsmoral krankt, ist Erfahrung gefragt. Wir sind die Spezialisten, entlasten Sie von Inkassosorgen und treiben für Sie die Honorarforderungen ein.

Und all dies zu fairen Konditionen, mit grosser Sensibilität und mit der gefragten Kompetenz. Damit Sie sich aufs Wesentliche konzentrieren können. Zum Wohl Ihrer Patientinnen und Patienten.

\section{Praxisübernahme}

Oft wechseln Occasionsröntgenanlagen, z.B. auch bei Praxisübernahmen, ihren Besitzer. Vor der Übernahme sollten folgende Punkte geprüft werden:

- Entspricht die Anlage den BAG-Vorschriften? Ein Blick ins Anlagebuch schafft Klarheit.

- Erfüllt die Anlage die Vorschriften auch nach dem 1. Januar 2008?

- Ist die Anlage fachmännisch revidiert worden?

- Sind noch Ersatzteile lieferbar?

- Gewährt der Verkäufer noch eine Garantiezeit?

- Ist die Röntgenröhre in gutem Zustand?

- Erfüllt das Stativ Ihre Anforderungen? Höhenverstellung, variabler Film/Fokus-Abstand, bewegliches Raster...

- Ist bei einem Umzug im Preis auch die ganze Arbeit inbegriffen? Demontage, Montage, Abnahmeprüfungen, ggf. auch Entsorgungsgebühren.

Es empfiehlt sich, unbedingt eine Zweitmeinung einzuholen. Die Merkblätter des BAG zur ionisierender Strahlung können Ihnen in vielen Fällen weiterhelfen (www.bag.admin.ch).

\section{Votre partenaire pour le règlement de vos factures!}

Avez-vous des patients mauvais payeurs? Si cela vous irrite, faites appel à notre expérience. Nous sommes des spécialistes, nous nous occupons de vos soucis de contentieux et encaissons pour vous les honoraires dus!

Et tout ceci à des conditions équitables, avec tact et compétence. Pour que vous puissiez vous concentrer sur l'essentiel. Pour le bien des vos patients et patientes.

FMH Inkasso Services

Thorackerstrasse 3, 3074 Muri b. Bern Tel. 03195080 30, Fax 0319508040

E-Mail: mail@fmhinkasso.ch 